\title{
Image Denoising based on MAP Estimation using Dual Tree Complex Wavelet Transform
}

\author{
M Ramanjaneya Rao \\ M.Tech Student, \\ Gudlavalleru Engineering College, Gudlavalleru, \\ Krishna Dist, A.P., India, Pincode-521356
}

\author{
P Ramakrishna \\ Assistant Professor \\ Gudlavalleru Engineering College, Gudlavalleru \\ Krishna Dist, A.P., India, Pincode-521356
}

\begin{abstract}
Wavelet shrinkage is a standard technique for image denoising. Using the good directionality and shift invariance properties of dual tree complex wavelet transform, a new algorithm for image denoisinig is proposed. In this algorithm, the decomposed coefficients combined with the bivariate shrinkage model for the estimation of coefficients in high frequency sub bands and Bayesian shrinkage method is applied in order to remove the noise in highest frequency subband coefficients. The experimental results are compared with the existing shrinkage methods Visu and Bayes shrinkage methods in terms of peak signal-to-noise ratio (PSNR) and structural similarity index (SSIM).
\end{abstract}

\section{Keywords}

Dual Tree Complex wavelet transform (DTCWT), Bivariate shrinkage, Bayes shrinkage, Peak signal to noise ratio (PSNR), Structural similarity index (SSIM)

\section{INTRODUCTION}

In image processing it is a common problem to denoising the natural images corrupted by Gaussian noise. Denoising is a technique that removes the added noise in the original input signal or image. It is impossible that completely recover an exact representation of original input data when observed in combination with added noise. However, a good representation of the original input data must be retaining as much as possible by using the denoising techniques.

Over the last few decades there are many different techniques have been developed for denoising. These techniques are explained in [1]. Wavelets are the one of those techniques used for removing of noise introduced in original data. Discrete wavelet transform is the most widely used technique. Denoising algorithm using wavelet transform is

1. Decompose the image using analysis filter bank.

2. Modify the coefficients according to some threshold rule.

3. Apply the synthesis filter bank to get denoised image.

There are two problems with discrete wavelet transform, shift invariance and data directionality [2-4]. The two problems can be overcome by using dual tree complex wavelet transform.

Denoising of images using wavelets is based on coefficient shrinkage i.e. reduction of transform coefficients. The coefficients are modified according to some threshold value using different threshold functions. The threshold value is estimated by using thresholding rules. Various thresholding rules like Visu, universal and sure shrink are explained in [56], MAP estimation based shrinkage rules i.e. Bayes and bivariate shrinkage functions are explained in [5-7]. A new shrinkage method is proposed using DT-CWT in [2].
The remaining of this paper is organized as follows; section II explains the concept Dual tree complex wavelet transform. Section III explains the concept of MAP estimation based shrinkage rules. Section IV explains the proposed image denoising algorithm. Section V presents the experimental results and analysis and section $\mathrm{V}$ draws the final conclusion.

\section{DUAL TREE COMPLEX WAVELET TRANSFORM}

Kingsbury of Cambridge University proposed the dual-tree complex wavelet transform in 1998. The dual tree complex wavelet transform has two sets of parallel real DWTs. The upper DWT represents the real part of DT-CWT and the lower DWT represents the imaginary part of DT-CWT [3]. The figure 1 shows the analysis filter bank for Dual Tree Complex Wavelet Transform (forward DT-CWT), $\mathrm{h}_{0}$ is the low-pass filter and $h_{1}$ is the high-pass filter of real part, $g_{0}$ is the lowpass filter and $g_{1}$ is the high-pass filter of imaginary part. The DT-CWT uses two different sets of real filters to generate wavelet coefficients separately for the real part and imaginary part.

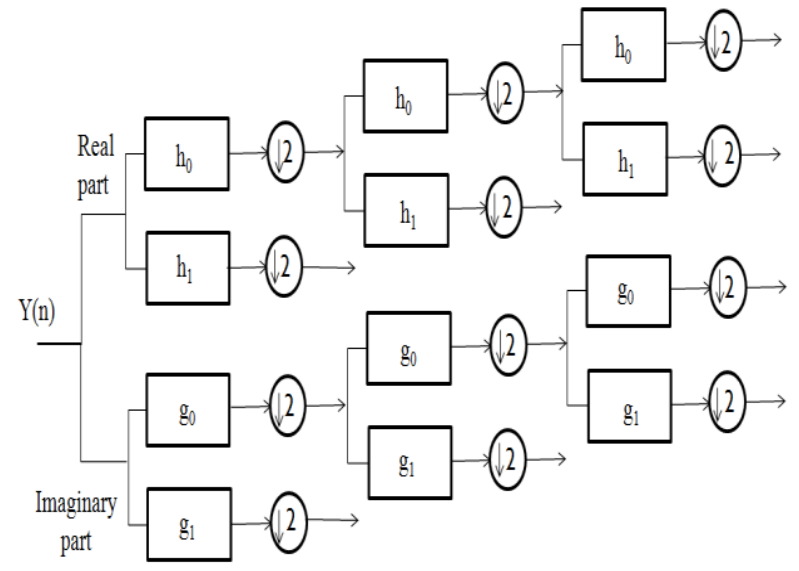

Fig 1: Analysis filter bank for 1-D Dual Tree Complex Wavelet Transform

The two dimension (2-D) dual-tree complex wavelet transform is obtained by the tensor of one dimension transform, which is similar with 2-D wavelet transform. The 2-D DT-CWT provides the image details and orientations, which is different from the 2-D wavelet transform. Therefore, the dual-tree complex wavelet transform has good directional selectivity. The figure 2 illustrates the six orientations of 2-D DT-CWT. 


\section{DT-CWT REAL PART}

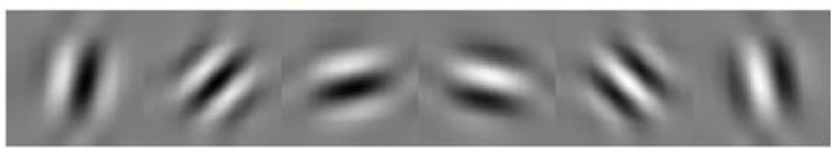

DT-CWT IMAGINARY PART

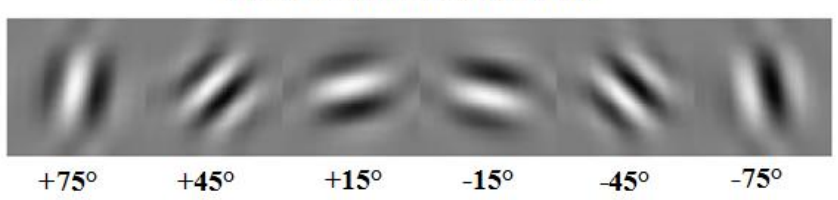

Fig 2: Six orientations of DT-CWT

The denoised image can be reconstructed from the Inverse Dual Tree Complex Wavelet Transform. Figure 3 shows synthesis filter bank for dual tree complex wavelet transform which is constructed with set of high pass and low pass filters. The synthesis filter bank forms approximately Hilbert transform pair with the analysis filter bank.

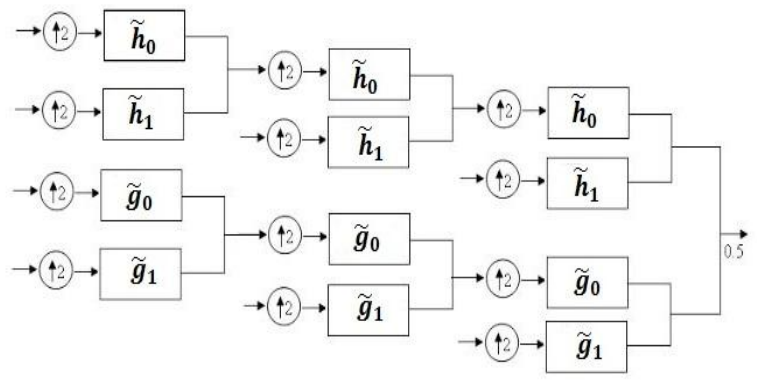

Fig. 3 Synthesis filter bank for DT-CWT

\section{SRINKAGE RULES}

Donoho and Johnston proposed Visu shrink by applying universal threshold, Visu Shrink is found to yield a highly smoothed estimate [8]. SURE shrink is the combination of universal threshold and SURE threshold was developed by Donoho and Johnston.

\subsection{Bivariate Shrinkage Model}

In bivariate model, the Bayesian estimation problem is modified by taking account in the statistical dependency between coefficient and parent [7]. It is a shrinkage function depends upon both coefficient and its parent value. The Bivariate model is proposed at the point of the relativity of coefficients at different scales. The joint probability distribution was established by bivariate model of child and parent coefficients and the coefficients are estimated by using MAP estimation.

In wavelets, after decomposition of input image the coefficients are represented as follows

$$
y=w+n
$$

Where ' $y$ ' is the noisy wavelet coefficient, ' $w$ ' is the original coefficient and ' $n$ ' is the Gaussian noise. Image denoising is a process that estimating the true wavelet coefficient by observing the noise.

Let us consider the wavelet coefficient w2 be the parent of wavelet coefficient $w 1$ (w2 is the wavelet coefficient at the same position as $w 1$, but at next scale). Then

$$
y_{1}=w_{1}+n_{1}
$$

$$
y_{2}=w_{2}+n_{2}
$$

Where $y_{1}$ and $y_{2}$ are the observed noisy wavelet coefficients and $\mathrm{w}_{1}$ and $\mathrm{w}_{2}$ true wavelet coefficients. By converting these two above equations into vector form, then

$$
y=w+n
$$

Where $\mathrm{y}=\left[\mathrm{y}_{1}, \mathrm{y}_{2}\right], \mathrm{w}=\left[\mathrm{w}_{1}, \mathrm{w}_{2}\right]$ and $\mathrm{n}=\left[\mathrm{n}_{1}, \mathrm{n}_{2}\right]$.

Now applying the standard Maximum A Posteriori estimator for ' $w$ ', then the estimation for true wavelet coefficients is given by

$$
\widehat{w}_{M A P}(y)=\operatorname{argmax}_{w}\left[p_{w / y}(w / y)\right]
$$

Using Bayes rule, the above equation is modified as

$$
\begin{aligned}
\widehat{w}_{M A P}(y) & =\operatorname{argmax}_{w}\left[p_{y / w}(y / w) \cdot p_{w}(w)\right] \\
& =\operatorname{argmax}_{w}\left[p_{n}(y-w) \cdot p_{w}(w)\right]
\end{aligned}
$$

According to the assumption, the noise is independent and identically distributed Gaussian, the joint probability distribution function of noise coefficients are achieved by

$$
p_{n}(n)=\frac{1}{2 \pi \sigma_{n}^{2}} \cdot \exp \left(-\frac{n_{1}^{2}+n_{2}^{2}}{2 \sigma_{n}^{2}}\right)
$$

The joint probability distribution function of wavelet coefficients are achieved by

$$
p_{w}(w)=\frac{3}{2 \pi \sigma_{1} \sigma_{2}} \cdot \exp \left(-\sqrt{3} \sqrt{\left(\frac{w_{1}}{\sigma_{1}}\right)^{2}+\left(\frac{w_{2}}{\sigma_{2}}\right)^{2}}\right)
$$

The solution is

$$
\widehat{w}_{1}=\frac{\left(y_{1}^{2}+y_{2}^{2}-\frac{\sqrt{3} \sigma_{n}^{2}}{\sigma}\right)_{+}}{\sqrt{y_{1}^{2}+y_{2}^{2}}} \cdot y_{1}
$$

Where $\sigma_{\mathrm{n}}^{2}$ is the noise variance and calculated using the equation (2) and $\sigma$ is the signal variance without noise.

$$
\begin{gathered}
\hat{\sigma}_{n}{ }^{2}=\frac{\operatorname{median}\left(\left|y_{i}\right|\right)}{0.6745} \\
\sigma=\max \left(\hat{\sigma}_{y}{ }^{2}-\hat{\sigma}_{n}{ }^{2}, 0\right)
\end{gathered}
$$

The above equation (12) shows the expression for bivariate shrinkage function.

\subsection{Bayes threshold}

Bayes Shrink was proposed by Chang, $\mathrm{Yu}$ and Vetterli. The Bayesian threshold is defined [7] as

$$
\lambda=r \times \sigma_{n}{ }^{2} / \sigma_{x}
$$

Where ' $r$ ' is the flexible parameter and is equal to $\sqrt{2}$ for superior denoising effect. $\sigma_{\mathrm{n}}$ is the noise variance and calculated by using median estimation and $\sigma_{\mathrm{x}}$ is the input image variance and calculated using the following equation

$$
\sigma_{x}^{2}=\operatorname{var}(y)-\hat{\sigma}_{n}^{2}
$$

For estimation of noise free coefficients the threshold estimation methods are combined with the threshold filters like hard and soft threshold. 
The hard threshold function was given

$$
H T=\operatorname{Hard}(c)=\left(\begin{array}{ll}
c, & \text { for }|c|>\lambda \\
0, & \text { for }|c| \leq \lambda
\end{array}\right)
$$

Similarly for soft thresholding function

$$
S T=\operatorname{Soft}(c)=\left(\begin{array}{ll}
\operatorname{sign}(c) \max ((|c|-\lambda), 0), & \text { for }|c|>\lambda \\
c, & \text { for }|c| \leq \lambda
\end{array}\right)
$$

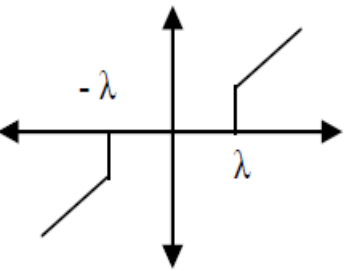

(a)

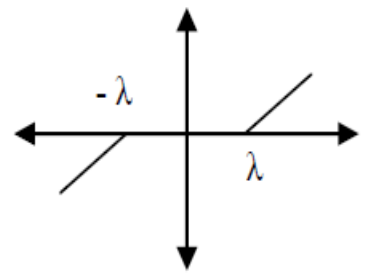

(b)
Fig 4: (a) Hard threshold function, (b) Soft threshold function

\section{PROPOSED ALGORITHM}

The bivariate shrinkage rule is based on parent and child coefficients. At the highest level the parent coefficients are absent. So, the bivariate shrinkage rule cannot be estimate the shrinkage coefficients at the last level. In order to remove the noise present at last scale, Bayesian shrinkage is used.

The algorithm is given as follows

Input $=$ Digital image

Output $=$ Denoised Image

1) Read the input image

2) Add noise to the input image

3) Decompose the input image using Dual Tree Complex Wavelet Transform and set the number of stages to 4

4) Estimate the high frequency sub-band coefficients from level 1 to level 3

a) Estimate the noise variance using median estimator using equation (10)

b) Determine the original wavelet coefficients without noise every sub-band using equation (11)

5) Using equation (9) estimate the original coefficients

6) Using Bayesian threshold estimation method, estimate the highest frequency sub-band coefficients at level 4.

a) Estimate the noise variance of highest frequency sub-band using equation (10)

b) Determine the original coefficients variance using equation (13)

c) Estimate the threshold $\lambda$ by using equation (12), develop the highest frequency coefficients using soft/hard thresholding function.

7) Finally apply the inverse dual tree complex wavelet transform and extract the denoised version of input image.

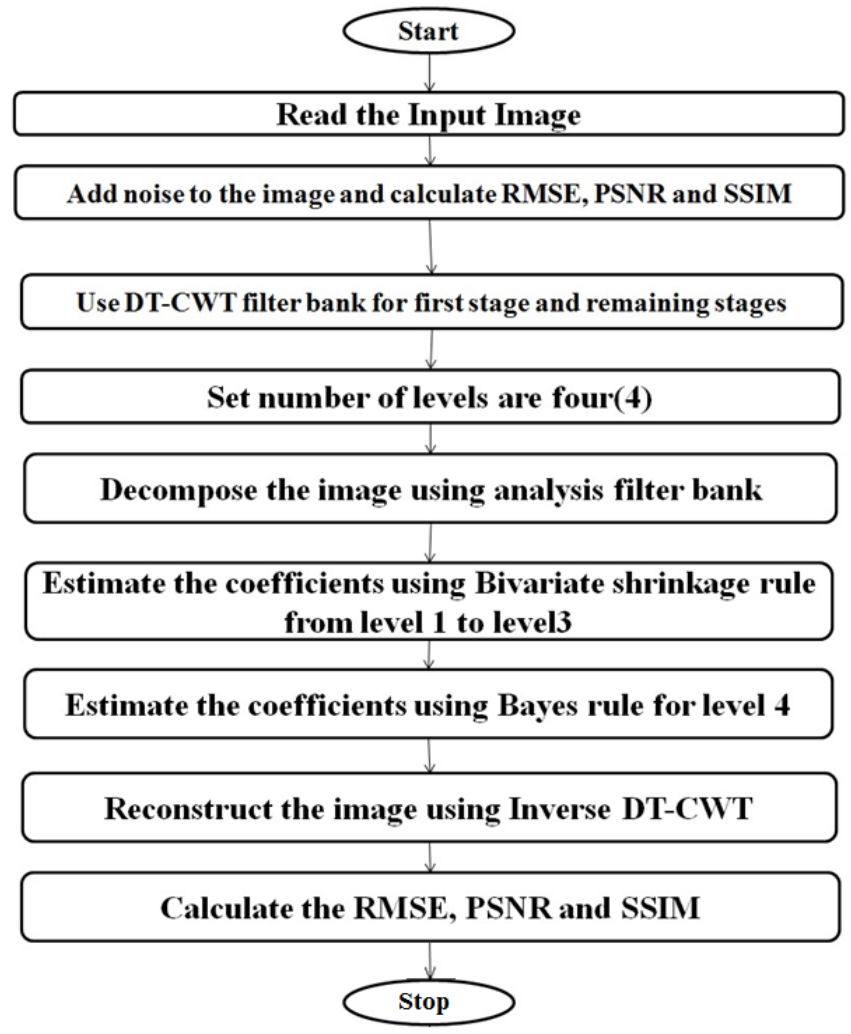

Fig 5: Flowchart of proposed algorithm

Using the shift invariance and good data directionality properties of the DT-CWT, the noisy input image is applied to the transform. The image is decomposed into detailed coefficients $\left(\mathrm{d}_{\mathrm{m}, \mathrm{n}}^{\mathrm{j} j}\right)$ and approximate coefficients $\left(\mathrm{C}_{\mathrm{m}, \mathrm{n}}\right)$. The approximate coefficients are also known as scalar coefficients which are from the low pass filter. The detailed coefficients are also known as wavelet coefficients which are from the high pass filter. The detailed coefficients are mixed with the bivariate shrinkage model from level 1 to level 3 to estimate the noise free coefficients. At the level 4 i.e. last level, the coefficients don't have the parent values. So, the coefficients cannot be estimated by bivariate shrinkage method. Bayesian shrinkage method used to remove the noise present at level 4 with hard or soft threshold functions. The shrinked coefficients are applied to the inverse wavelet transform to get denoised version of input noisy image. Estimation of image using the dual tree complex wavelet transform is summarized by the following equation.

$$
\begin{aligned}
& y \stackrel{\text { DTCWT }}{\longrightarrow} d=\left(c_{m, n}, d_{m, n}^{o, j}\right) \stackrel{\text { MAP ESTIMATOR }}{\longrightarrow} \\
& \longrightarrow\left\{c_{m, n}, \hat{S}_{m, n}^{o, j}=\delta\left(d_{m, n}^{o, j}\right)\right\} \stackrel{\text { IDTCWT }}{\longrightarrow} \hat{x}(t)
\end{aligned}
$$

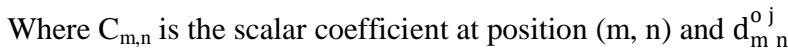
is the wavelet coefficient at position $(\mathrm{m}, \mathrm{n})$, orientation $\mathrm{o}$ and scale $\mathrm{j}$. The wavelet coefficients are estimated according to the proposed MAP estimation shrinkage algorithm. $\widehat{s}_{m ~}^{o j}$ is the shrinkage coefficient according to the proposed algorithm and $\delta$ is the thresholding operator.

In this paper, the output results of the proposed algorithm are compared with the traditional denoised methods of dual tree complex wavelet transform using Visu shrink and Bayes shrinkage in terms of peak signal to noise ratio and structural similarity index. 


\section{RESULTS AND ANALISYS}

To verify the performance of proposed method, firstly the proposed method is simulated with various images like Lena, Barbara and tooth etc., with different threshold estimation methods like Visu and Bayes shrinkage methods with noise variances 15, 20 and 30 with threshold filters soft and hard filters. Here the performance of the proposed method is compared with the parameters of peak signal to noise ratio (PSNR) and Structural Similarity index (SSIM) as follows

$$
\text { PSNR }=20 * \log \left(\frac{255}{\sqrt{\mathrm{MSE}}}\right)
$$

SSIM is a new approach that measures the quality of an image. SSIM was developed by Wang et.al [9]. Structural similarity index measures the quality between two images $X$ an $\mathrm{Y}$ as follows

$$
\operatorname{SSIM}(x, y)=\frac{\left(2 \mu_{x} \mu_{y} c_{1}\right)\left(2 \sigma_{x y}+c_{2}\right)}{\left(\mu_{x}^{2}+\mu_{y}^{2}+c_{1}\right)\left(\sigma_{x}^{2}+\sigma_{y}^{2}+c_{2}\right)}
$$

Where $\mu_{\mathrm{x}}$ and $\mu_{\mathrm{y}}$ are the means of image $\mathrm{X}$ and image $\mathrm{Y}$ respectively, $\sigma_{\mathrm{x}}{ }^{2}$ and ${\sigma_{\mathrm{y}}}^{2}$ are the standard deviations and $\sigma_{\mathrm{xy}}$ is the covariance of two images $\mathrm{X}$ and $\mathrm{Y}$ respectively. $\mathrm{C} 1$ and $\mathrm{C} 2$ are the constants [9].

Table 1: Denoising results for Lena image of size $512 \times 512$ with hard threshold filter

\begin{tabular}{|c|c|c|c|c|c|c|}
\hline $\begin{array}{c}\text { Standard } \\
\text { deviation }\end{array}$ & \multicolumn{2}{|c|}{$\sigma=15$} & \multicolumn{2}{c|}{$\sigma=20$} & \multicolumn{2}{c|}{} \\
\hline Parameter & PSNR & SSIM & PSNR & SSIM & PSNR & SSIM \\
\hline Noisy image & 24.59 & 0.9845 & 22.13 & 0.9718 & 18.59 & 0.9382 \\
\hline Visu shrink & 27.76 & 0.9908 & 27.08 & 0.9892 & 26.30 & 0.9873 \\
\hline Bayes & 26.84 & 0.9891 & 26.68 & 0.9888 & 26.62 & 0.9886 \\
\hline Proposed & 30.22 & 0.9954 & 29.62 & 0.9946 & 28.57 & 0.9933 \\
\hline
\end{tabular}

Table 2: Denoising results for Peppers image of size 256x256 with hard threshold filter

\begin{tabular}{|c|c|c|c|c|c|c|}
\hline $\begin{array}{c}\text { Standard } \\
\text { deviation }\end{array}$ & \multicolumn{2}{|c|}{$\sigma=15$} & \multicolumn{2}{c|}{$\sigma=20$} & \multicolumn{2}{c|}{$\sigma=30$} \\
\hline Parameter & PSNR & SSIM & PSNR & SSIM & PSNR & SSIM \\
\hline Noisy image & 24.60 & 0.9210 & 22.14 & 0.8952 & 18.55 & 0.8456 \\
\hline Visu shrink & 20.70 & 0.9273 & 20.29 & 0.9243 & 19.95 & 0.9252 \\
\hline Bayes & 22.72 & 0.9466 & 22.44 & 0.9437 & 22.69 & 0.9444 \\
\hline Proposed & 27.15 & 0.9587 & 26.91 & 0.9575 & 24.86 & 0.9522 \\
\hline
\end{tabular}

Table 3: Denoising results for Barbara image of size 256x256 with soft threshold filter

\begin{tabular}{|c|c|c|c|c|c|c|}
\hline $\begin{array}{c}\text { Standard } \\
\text { deviation }\end{array}$ & \multicolumn{2}{|c|}{$\sigma=15$} & \multicolumn{2}{c|}{$\sigma=20$} & \multicolumn{2}{c|}{$\sigma=30$} \\
\hline Parameter & PSNR & SSIM & PSNR & SSIM & PSNR & SSIM \\
\hline Noisy image & 24.55 & 0.9761 & 22.11 & 0.9583 & 18.56 & 0.9133 \\
\hline Visu shrink & 19.41 & 0.9588 & 19.68 & 0.9582 & 19.53 & 0.9573 \\
\hline Bayes & 21.52 & 0.9728 & 21.47 & 0.9725 & 20.80 & 0.9681 \\
\hline Proposed & 26.41 & 0.9893 & 25.52 & 0.9872 & 24.67 & 0.9854 \\
\hline
\end{tabular}


The tables $1 \& 2$ represents the comparison of the denoising results of Lena and Peppers images of sizes $512 \times 512$ and $256 \times 256$ respectively with the parameters of Peak signal to noise ratio and structural similarity index. The compared results with Bayes shrinkage and Visu shrinkage shows that the proposed algorithm gives better results. The table 3 shows the comparison of denoising results of Barbara images of size 256X256 with the parameters PSNR and SSIM. The compared results show the proposed shrinkage algorithm provides better results.

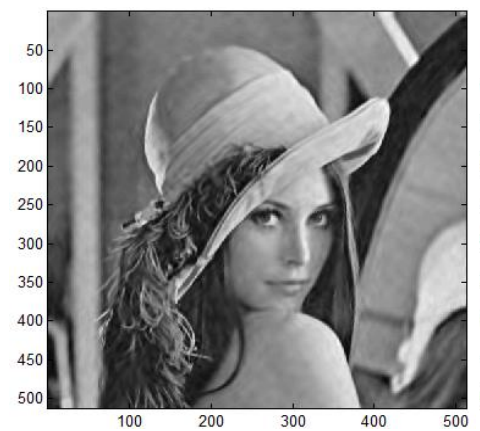

(a)

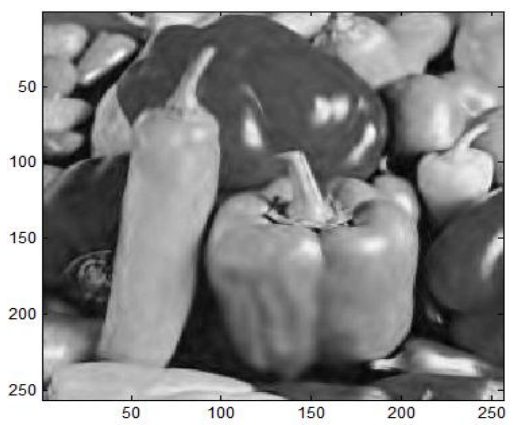

(d)

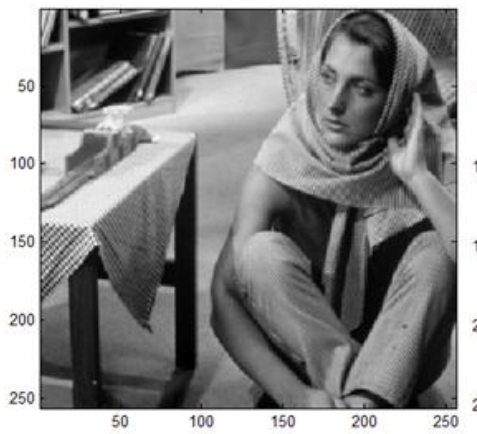

(g)

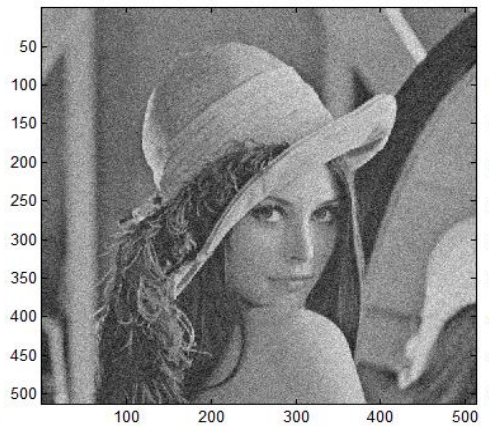

(b)

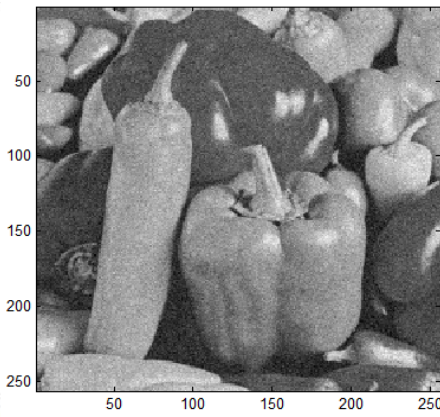

(e)

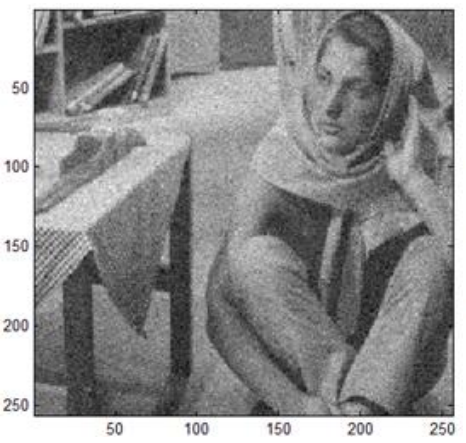

(h)

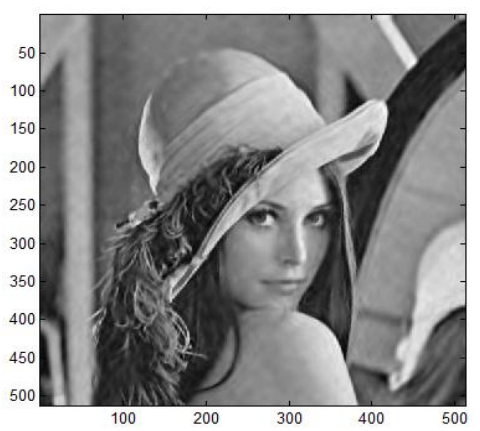

(c)

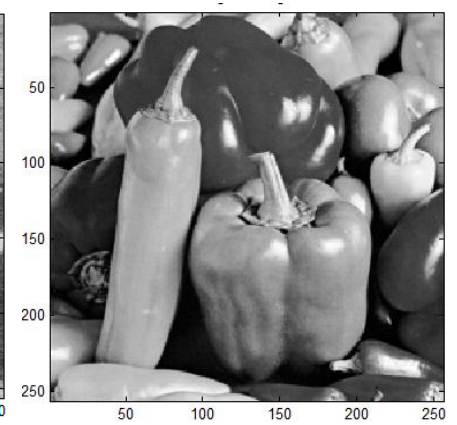

(f)

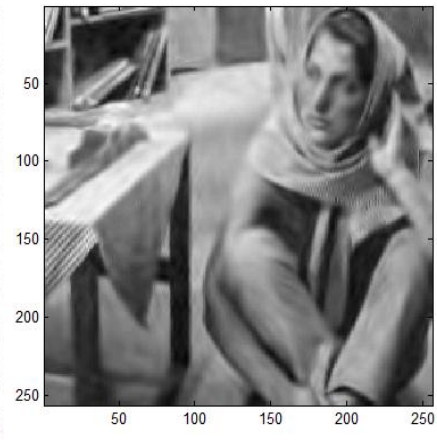

(i)

Fig 6: Denoising results for proposed algorithm (a) Lena, Original image (b) Lena, Noisy image ( $\sigma=30)$ (c) Lena, Denoised image (d) Peppers, Original image (e) Peppers, Noisy image $(\sigma=15)$ (f) Peppers, Denoised image (g) Barbara, Original image (h) Barbara, Noisy image $(\sigma=20)(i)$ Barbara, Denoised image.

Figure 6 shows the denoising results for different images with different noise variances. The experimental results show that the proposed method performs better than the traditional shrinkage methods like Visu shrink and Bayes shrink.

\section{CONCLUSION}

In this paper, a new shrinkage algorithm is proposed that effectively reduces the noise in images. The experimental outcome shows that the proposed technique can eliminate the noise efficiently and protect the edge information well, this algorithm provides good denoising effect as well as peak signal to noise ratio and structural similarity index is better than traditional denoising methods uses soft and hard threshold methods. The Dual-Tree Complex Wavelet Transform not only has the good aspect of traditional wavelet transform but also provides good directional selectivity and shift invariance. In future this proposed technique can be applied to different types of wavelets and this proposed technique can applied to color images and videos also.

\section{ACKNOWLEDGMENT}

The authors place on record their thanks to the authorities of Gudlavalleru Engineering College, A.P for the facilities they provided

\section{REFERENCES}

[1] Survey of Image Denoising Techniques by Mukesh C. Motwani, Mukesh C. Gadiya, Rakhi C. Motwani, Frederick C. Harris, Jr. 
[2] Massimo Fierro, Ho-Gun Ha, and Yeong-Ho Ha, "Noise Reduction Based on Partial Reference, Dual-Tree Complex Wavelet Transform Shrinkage”, IEEE Transactions on Image Processing, vol. 22, no. 5, pp. 1859-1872, 2013.

[3] W. Selesnick, R. G. Baraniuk, and N. G. Kingsbury, - The dual-tree complex wavelet transform-A coherent framework for multiscale signal and image processing, \| IEEE Signal Processing. Mag., vol. 22, no. 6, pp. 123151, Nov. 2005.

[4] Mr. R. K. Sarawale, Dr. Mrs. S.R. Chougule, “ Image denoising using dual-tree complex dwt and doubledensity dual-tree complex dwt", International Journal of Advanced Research in Computer Engineering \& Technology (IJARCET) Volume 2, Issue 6, June 2013.

[5] Rajesh Kumar Rai , Jyoti Asnani and T. R. Sontakke, "Review of Shrinkage Techniques for Image Denoising", International Journal of Computer Applications (0975 8887) Volume 42- No.19, March 2012.

[6] Sachin D Ruikar and Dharmpal D Doye, "Wavelet Based Image Denoising Technique", International Journal of Advanced Computer Science and Applications (IJACSA), Vol. 2, No.3, March 2011.Levent S,endur, and Ivan W. Selesnick, "Bivariate Shrinkage Functions for Wavelet-Based Denoising Exploiting Interscale Dependency", IEEE Transactions On Signal Processing, Vol. 50, No. 11, pp. 2744-2756, November 2002.

[7] Levent S,endur, and Ivan W. Selesnick, "Bivariate Shrinkage Functions for Wavelet-Based Denoising Exploiting Interscale Dependency", IEEE Transactions On Signal Processing, Vol. 50, No. 11, pp. 2744-2756, November 2002.
[8] Complex wavelet transforms and their applications, M.Phill thesis by Panchamkumar D Shukla.

[9] Yusra A. Y. Al-Najjar, Dr. Der Chen Soong, "Comparison of Image Quality Assessment: PSNR, HVS, SSIM, UIQI", International Journal of Scientific \& Engineering Research, Volume 3, Issue 8, August-2012.

[10] B.Chinnarao, M.Madhavilatha , "Improved Image De noising Algorithm using Dual Tree Complex Wavelet Transform", International Journal of Computer Applications (0975 - 8887) Volume 44- No20, April 2012 .

[11] Devanand Bhonsle, Sandeepa Dewangan, "Comparative Study of dual-tree complex wavelet transform and double density complex wavelet transform for Image Denoising Using Wavelet-Domain" International Journal of Scientific and Research Publications, vol. 2, Issue 7, pp. 1-5,July 2012.

[12] Image denoising using dual tree statistical models for complex wavelet transform coefficient magnitudes by P.R.Hill, A.Achin, D.R.Bull and M.E. Al-Mualla.

[13] Mrs. Ritu Chouhan, Prof. Vikas Gupta, Arpita Rani Vaishnava, "Wavelet Based Color Image Denoising through a Bivariate Pearson Distribution", International Journal on Recent and Innovation Trends in Computing and Communication ISSN 2321 - 8169 Volume: 1 Issue: 4, April 2013.

[14] Rudra Pratap Singh Chauhan, Sanjay Singh and Sanjeev Kumar Shah, "A Practical Approach of Complex Dual Tree DWT for Image Quality Improvement and Denoising", International Journal of Modern Engineering Research (IJMER) Vol.1, Issue.2, pp-632-636. 